\title{
RELACIÓN ENTRE DECISIONES ARBITRALES Y VARIABLES DEPORTIVAS EN EL FÚTBOL PROFESIONAL DE COSTA RICA
}

\author{
María Clara Rodríguez y Walter Salazar Rojas \\ Escuela de Educación Física y Deportes \\ Universidad de Costa Rica, San José, Costa Rica \\ E-mail:wsalazar@cariari.ucr.ac.cr
}

\begin{abstract}
Resumen
Rodríguez Salazar, M. C. y Salazar Rojas, W. (2002). Relación entre decisiones arbitrales y variables deportivas en el fútbol profesional de Costa Rica. Revista de Ciencias del Ejercicio y la Salud, 2(2), 5061. Con el objetivo de determinar objetivamente si existen algunas tendencias en los juicios arbitrales del fútbol costarricense, específicamente en cuanto a amonestaciones y expulsiones, se analizaron 575 partidos de primera y segunda división jugados durante los años 2001 y 2002, a partir de los informes arbitrales oficiales. Se confirmó la hipótesis de la ventaja para el equipo local, así como la tendencia a compensar las sanciones entre los equipos contrincantes, especialmente con respecto a las expulsiones; no hubo diferencias en función del nivel de los árbitros, ni se evidenciaron sesgos en función de los equipos. Se hallaron diferencias significativas individuales entre los árbitros. También se encontraron diferencias significativas para tarjetas rojas (TA) y tarjetas amarillas (TR) entre primer y segundo tiempo, y con relación al número total de goles marcados. En conclusión, se observó mayor discriminación en las decisiones arbitrales frente a las TR y mayor homogeneidad frente a las TA.

Palabras clave: Toma de decisiones, juicios arbitrales, desempeño arbitral
\end{abstract}

\section{INTRODUCCIÓN}

La función del árbitro es esencial para que exista el fenómeno deportivo; sin árbitros no hay competencia. En el fútbol específicamente, los árbitros ganan cada vez mayor protagonismo pero mas por sus errores que por sus aciertos, los cuales llegan a incidir sobre decisiones posteriores trascendentales. El arbitraje supone una tarea de naturaleza cognitiva en tanto se basa en procesar información para emitir un juicio. De hecho, el proceso de toma de decisiones y el juicio resultante se ha constituido recientemente en una línea de investigación dentro del arbitraje deportivo. La $17^{\text {th }}$ Research Conference on Subjective Probability, Utility and Decision Making, con apoyo de La European Association for Decision Making (EADM) ofrece una visión general sobre el estado del conocimiento respecto al proceso de juicios y toma de decisiones. Según Roelofsma y Van der Pligt (2001), en los últimos 10 años esta área se ha orientado al estudio del tiempo de reacción electiva. En Europa existe una fuerte línea de investigación tendiente a incorporar el tiempo en una estructura teórica diferente que permita mejorar la comprensión del juicio y la toma de decisiones. Estos autores afirman que de acuerdo con varias investigaciones, el comportamiento de elección cambia dramáticamente al introducir incertidumbre en la representación de opciones

Los estudios sobre juicios y toma de decisiones basados en la teoría de las probabilidades, suelen referir a los sujetos a situaciones en las que la incertidumbre sobre un resultado depende de fuentes externas a su decisión, pero en otros casos la incertidumbre enfrentada para la toma de decisiones depende de fuentes internas (conocimiento, información). De acuerdo con Bereby-Mayer, Meyer y Budesco (2003), las leyes de probabilidad son mas factibles en las situaciones en las que la incertidumbre es externa que cuando es interna. En una tarea de elección múltiple, el sujeto debe decidir entre dar respuesta o no (omitir) frente a un estímulo con base en el conocimiento que el sujeto posee en ese momento. La estrategia óptima depende de la recompensa asociada con esas dos opciones según la regla de puntuación 
establecida. Cuando la omisión no implica consecuencia negativa (regla positiva) aunque no se posea información, la tendencia a responder es mayor, que cuando la consecuencia de la omisión es negativa (regla negativa). Así el tomar una decisión requiere evaluar el valor esperado asociado tanto a la respuesta como a la no respuesta; el punto clave es la probabilidad subjetiva del actor de que su opción es la correcta, lo que significa una situación de incertidumbre interna. Mediante la manipulación experimental de la regla de puntuación (positiva o negativa) en situaciones de elección múltiple, los autores encontraron una tasa diferencial de omisiones que sigue el patrón predicho por la teoría de las probabilidades: los sujetos presentaron mayor número de omisiones en las situaciones de regla positiva que en las de regla negativa.

Cuando se le pide a un sujeto realizar una tarea bajo condiciones de incertidumbre su juicio por lo general está influido por la expectativa, concepto utilizado para explicar un amplio rango de tareas de tiempo de reacción (TR). Jentzsch y Sommer (2002) realizaron un estudio sobre tareas de TR manipulando la expectativa intencional; hallaron que el TR es mas corto para eventos esperados que para los no esperados. Sin embargo, el concepto de expectativa no es un fenómeno unitario, puede consistir de diferentes subtipos. Formas pasivas de expectativas se consideran mas automáticas y fáciles mientras que las formas activas conllevan un nivel de capacidad atencional. Se considera que las dos trabajan independientemente y pueden mostrar efectos simultáneos sobre la ejecución.

Otro elemento estudiado con respecto al proceso de toma de decisiones, es el efecto que la decisión tomada tiene sobre el actor. Según Zeelemberg, Van der Pligt y De Vries (2000), el grado en el que una persona se siente mejor o peor después de tomada una decisión no solo depende del resultado de su decisión, también depende de cómo el resultado es alcanzado; resultados logrados a través de la acción generalmente conllevan una reacción afectiva mas intensa que cuando los mismos resultados son logrados por la omisión. Los autores emplean el término "efecto del actor" que diferencian de la tendencia a la omisión en tanto esta se refiere además de la respuesta afectiva, al componente moral e intencional de la elección. Las personas pueden anticipar la reacción postdecisión y considerarla al momento de tomar la decisión. De acuerdo con la teoría atribucional, la respuesta afectiva al éxito o al fracaso está en gran parte determinada por la atribución de responsabilidad. Así, se presume una relación entre el efecto de actor y los procesos atribucionales. Los autores comprobaron que la reacción afectiva es mas negativa hacia el actor activo (quien toma la decisión de manera deliberada) que hacia el actor pasivo (decisión asignada o decisión de no actuar), manteniendo constante el resultado obtenido; significa que la reacción afectiva siguiente al resultado de la decisión depende de procesos atribucionales, a mayor responsabilidad atribuida mayor la intensidad de la reacción afectiva postdecisión.

El estudio de la toma de decisiones dentro del contexto deportivo específicamente, ha sido menos estudiado a pesar de la cantidad de investigación en torno al tiempo de reacción. Alain, Sarrazin y Lacombre (1986) parten del concepto de que tanto la velocidad como la precisión en el procesamiento de información que tienen lugar después de ocurrido un estímulo, están influidos por el estado de preparación (disposición o respuesta anticipatoria) que el sujeto decide tener sobre el estímulo antes de que este ocurra. Los individuos parecen ser capaces de decidir conscientemente si deben orientar su preparación a favor de un evento o no, y si es así, en qué magnitud debe darse. Los autores afirman que numerosos experimentos de tiempo de reacción electiva y el efecto de diferentes factores (como resultado predicho, confianza de los sujetos en el resultado predicho, probabilidad de ocurrencia del estímulo, y tiempo límite para reaccionar), respaldan lo anterior. A pesar de que el estado de preparación ha recibido la mayor parte de la atención experimental, es poco lo que se conoce sobre la estrategia cognitiva que guía a los sujetos en sus elecciones de estados de preparación. En consecuencia, los autores llevaron a cabo un estudio experimental con 
jugadores de squash para medir tiempo de procesamiento y elección del sujeto del estado de preparación variando dos factores: probabilidad de ocurrencia y presión de tiempo manipulando la velocidad con la que era lanzada la bola a la que debían responder (lob o smash) y la probabilidad de ocurrencia de cada tipo de tiro la cual era informada al sujeto. Corroboraron lo hallado por estudios previos: el sujeto tiende a orientar su estado de preparación hacia el estímulo que presenta mayor probabilidad de ocurrencia debido a una mayor expectativa; comprobaron además que la elección del estado de preparación del ejecutante es el resultado de una valoración conjunta de la probabilidad de ocurrencia (valor esperado) y de la presión de tiempo.

El juicio deportivo implica dos aspectos: decidir si una posición, una situación o un objeto están permitidos por el reglamento, y valorar la ejecución del deportista de manera objetiva, tarea que resulta generalmente mas difícil y que frecuentemente es generadora de desacuerdos entre diferentes jueces, por lo que ha sido blanco de mayor estudio. Las primeras investigaciones sobre juicios deportivos estuvieron referidas a la gimnasia; la primera revisión data de 1970, realizada por Landers después de la cual se siguió la línea de trabajos guiada por Scheer. En uno de sus trabajos pioneros (Scheer, 1973), analizó las calificaciones otorgadas por jueces en función de la ubicación de los gimnastas en el orden de presentación, para lo que dividió a la muestra de gimnastas que participaron en una competencia universitaria en tres tercios, el inicial, el medio y el último; encontró que las calificaciones fueron mas altas para las gimnastas que se presentaron en el último tercio en comparación con las del tercio inicial y medio, en tres de los siete eventos: barra horizontal, trampolín y barras paralelas. Estos resultados fueron recientemente confirmados por Plessner (1999) quien también estudió el efecto de ubicación entre jueces de gimnasia pero experimentalmente y desde una aproximación social-cognitiva; comprobó que la expectativa de los jueces en función del orden de presentación influye en las calificaciones otorgadas a los atletas, de modo que las gimnastas cuando se presentaron hacia el final del evento obtuvieron valoraciones mas positivas que cuando lo hicieron al inicio. En otro estudio, Ansorge y Scheer (1987) identificaron un patrón de sesgo entre los jueces durante la realización de los Juegos Olímpicos de 1984; hallaron una tendencia generalizada que opera en dos vías entre los jueces internacionales: tendencia a favorecer a los gimnastas representantes de sus mismos países de origen, y tendencia a desfavorecer a los representantes de los países percibidos como los rivales más próximos a sus coterráneos.

De otro lado, en los deportes con oposición la figura del árbitro es más frecuente que la de juez por cuanto además de decidir la legalidad de una jugada según el reglamento, debe arbitrar la respectiva sanción, lo que implica tres tareas: percibir la situación, compararla con el reglamento o interpretarla, y sancionar el acto deportivo cuando corresponda (Caracuel, Andreu y Pérez, 1995). Es la interpretación la que exige del árbitro mayor competencia por cuanto debe valorar no solo la acción sino también la intención del acto. Es esta función la que se traduce en una fuente de estrés pues con frecuencia su interpretación o lectura de la intencionalidad del jugador no corresponde con la que hacen los aficionados, los periodistas o la misma dirigencia deportiva.

Dos variables ha sido reiteradamente estudiadas con relación al arbitraje deportivo: la experiencia y el fenómeno de la ventaja de local. Vasconcelos-Raposo y Carvalho (2000) encontraron que los árbitros de balonvolea con experiencia internacional muestran un mejor perfil psicológico en cuanto a ansiedad y autoconfianza en comparación con los más jóvenes e inexpertos. Segura y cols. (1999) mediante un trabajo descriptivo, no encontraron diferencias entre árbitros de baloncesto con alta y baja experiencia en cuanto a control de pensamiento en la toma de decisiones, aunque sugieren que la mayor experiencia los hace más sensibles para percibir conflictos. MacMahon y Ste-Marie (2002) utilizaron el paradigma de la experticia para examinar en dos estudios la toma de decisiones en árbitros de rugby: Midieron la detección de infracciones al reglamento y las fuentes de información empleadas En el primer 
estudio compararon las condiciones alta y baja experiencia; no hallaron diferencias respecto a la detección de señales pero sí en cuanto al uso de fuentes; los árbitros de mayor experiencia utilizaron mas fuentes de información que los de baja experiencia. En el segundo estudio compararon árbitros y jugadores; no hallaron diferencias significativas pero si una tendencia mayor a usar información de la memoria episódica para tomar decisiones entre los árbitros en comparación con los jugadores. Nevill, Balmer y Williams (2002) estudiaron la influencia tanto de la experiencia como del bullicio del público sobre las decisiones de árbitros de baloncesto, mediante el uso del video como técnica experimental; encontraron que al juzgar un partido en presencia del ruido de la gente, los jueces sancionaron menos faltas en contra del equipo local y sus juicios fueron más dudosos, en comparación con los partidos que observaron sin sonido, independientemente del nivel de experiencia; su explicación se basa en la tendencia del árbitro a evitar malestar con el público. Sin embargo, anteriormente Jones, Bray y Bolton (2001) realizaron un estudio tendiente a dilucidar si efectivamente es el público el responsable de la ventaja de localía. Para ello examinaron el comportamiento de árbitros de cricket a partir de los registros de partidos del English Club Cricket sobre el supuesto de que en tal deporte los espectadores además de ser pocos no constituyen fuente de presión; tomaron dos criterios: resultados y decisiones de expulsiones y no hallaron diferencias entre las decisiones de los árbitros a favor del equipo local con respecto del equipo visitante, mientras que sí hubo ventaja para el local en cuanto a juegos ganados; los autores concluyen que la tendencia a ganar cuando se compite en condición de local puede ser explicada por razones distintas a las decisiones arbitrales influidas por la presencia y presión de aficionados, tales como la familiaridad y comodidad que implica jugar en casa.

Específicamente con respecto al fútbol, también se ha demostrado que existe el fenómeno de la ventaja para el equipo que juega en condición de local. La Federación Internacional de Fútbol Asociado, FIFA, (2001) realizó un estudio sobre 6679 partidos internacionales jugados entre 1986 y 2000 para examinar el mito de la ventaja local desde una perspectiva netamente estadística y tomando como único criterio el resultado final. Encontraron que el equipo local gana el 50\% de los partidos mientras que el visitante solo lo hace el $25 \%$ de las veces, independientemente del nivel del campeonato que se juegue, de la importancia de los partidos; y de la región; observaron además que este fenómeno se presenta de manera mas acentuada en partidos de competición que en partidos amistosos. Por su parte, Nevill, Newell y Gale (1996) comprobaron también en la liga inglesa de fútbol la significativa ventaja en los resultados a favor de los equipos anfitriones.

Plessner y Betsch (2001) plantearon un nuevo factor a estudiar en relación con los juicios arbitrales; para ellos, la probabilidad de que un árbitro sancione una pena máxima en jugada dudosa es mayor si ante una falta similar previa del mismo equipo, no lo sancionó. Por ser esta una creencia generalizada, la sometieron a estudio experimental bajo el concepto de efecto de secuencia (ES), implicado en el modelo cognitivo. Sobre dicho concepto, Jentzsch y Sommer (2002) retomando trabajos sobre tiempo de reacción publicados dos décadas atrás, afirman que la toma de decisión ante una situación de tiempo de reacción electiva depende no solo de la probabilidad de ocurrencia del estímulo sino también del estímulo inmediatamente precedente el cual está relacionado con la expectativa subjetiva; definen dos tipos de ES clasificando cada evento como repetición o como alternativa del evento precedente: efecto de primer orden y efecto de orden superior según si se toman las consecuencias del evento inmediatamente precedente o del primer evento de la secuencia para el procesamiento del estímulo actual. Para su estudio, Plessner y Betsch utilizaron un video con 20 jugadas, 17 de tiro libre y tres de jugadas dudosas en área de pena máxima, dos sucesivas contra un equipo y la tercera contra el opuesto. 115 sujetos, árbitros y jugadores, fueron divididos en tres grupos: no observación de la primera jugada, sí observación y la sancionaron como pena máxima, y sí observación pero no la 
sancionaron como tal. De acuerdo con los resultados, la probabilidad de pitar un penal en jugada dudosa es mayor si ante una falta similar previa del mismo equipo, no fue sancionado; así mismo, el sancionar un penal incrementa la probabilidad de sancionar uno al equipo contrario; con respecto a las jugadas de tiro libro, las decisiones fueron independientes de las decisiones anteriores en situaciones similares. Comprobaron así que las decisiones pueden estar influidas por decisiones previas lo cual fue válido solo para los juicios que resultan determinantes como son las penas máximas. Los autores plantean la posibilidad de que después de una decisión de penal el árbitro experimenta un cambio de criterio, algo similar a lo que podría entenderse como una forma de compensación.

Otros juicios arbitrales que pueden ser decisivos durante el transcurso de un partido de fútbol además de las penas máximas, son las expulsiones. Son escasos los estudios específicos sobre tales sanciones disciplinarias. Torregrosa y Cruz (1997) estudiaron la influencia de los árbitros de fútbol en el comportamiento de juego limpio dentro del campo de juego mediante el análisis de 24 partidos de la Liga Profesional (LP) y de la Premier League (PL), grabados en video y codificados por tres expertos, según una plantilla de registro de comportamientos de fairplay. Encontraron que los árbitros sancionan más tarjetas amarillas al equipo que juega en condición de visitante que al que lo hace de local, mientras que no hallaron diferencias en el caso de las tarjetas rojas, adicionalmente hallaron mayor número de amonestaciones entre los partidos de la LP que en los de la PL. Jones, Paull y Erskine (2002) analizaron si el conocimiento del equipo y de los jugadores por parte del árbitro influye en sus juicios. Mediante la utilización de un video validado por expertos, conformaron dos grupos de árbitros; al grupo experimental le proporcionaron información previa sobre la negativa reputación de los jugadores de uno de los equipos mientras que al grupo control no le presentaron ninguna información; encontraron que el grupo experimental sancionó mayor número de tarjetas amarillas y tarjetas rojas contra el equipo sobre el que recibieron la información en comparación con el grupo control, lo que sugiere que el conocimiento previo influye sobre el comportamiento arbitral aunque es necesario ampliar estos hallazgos a la situación de campo.

En resumen, existe evidencia de que diferentes factores inherentes al desarrollo de un partido de fútbol pueden sesgar las decisiones que toman los árbitros durante el transcurso del mismo, especialmente las decisiones de mayor trascendencia. Simultáneamente, el público en general suele clasificar y calificar a los árbitros a partir de apreciaciones subjetivas que poco a poco se van consolidando como verdades colectivas, sin que exista hasta el presente un análisis objetivo que confirme la existencia de tendencias que orienten en una u otra dirección las decisiones que ellos toman. En consecuencia, el propósito del presente estudio fue determinar objetivamente las tendencias que se presentan en los juicios arbitrales del fútbol costarricense, específicamente en cuanto a amonestaciones y expulsiones.

\section{METODOLOGÍA}

\section{Sujetos}

La muestra estuvo constituida por los informes oficiales de los árbitros, correspondientes a 575 partidos jugados entre agosto de 2001 y marzo de 2003 de $1^{a}$ y $2^{a}$ divisiòn del fútbol de Costa Rica, informes que fueron facilitados por la Comisión de Arbitraje de la Federación Costarricense de Fútbol. No se incluyeron los partidos dirigidos por árbitros que habían actuado en menos de cinco ocasiones

\section{Instrumentos y procedimiento}

Una vez revisados los archivos de los informes arbitrales, se incorporó toda la información pertinente a una base de datos. Se eliminaron los informes incompletos o confusos y no se incluyeron aquellos que en el momento de hacer la revisión no estuvieron disponibles.

Las variables dependientes fueron el número de tarjetas amarillas (TA) y el número de tarjetas rojas (TR) sancionadas por los árbitros. En este último caso, no se discriminó entre expulsiones por doble amonestación o expulsión directa, dado que aunque la causa es 
diferente y no hace parte del interés de este estudio, el efecto es el mismo. La tercera variable dependiente, fue el índice de secuencia (I.S) para cuya obtención se procedió a seleccionar aquellos partidos en los que hubo al menos dos tarjetas para cada uno de los casos (TA o TR) de modo que permitiera aplicar el concepto de secuencia; posteriormente se identificó la secuencia temporal mediante la asignación de una $\mathrm{L}$ (local) o $\mathrm{V}$ (visitante) según el equipo objeto de la sanción; a continuación se asignó valor de 1 a cada secuencia alternada ( $\mathrm{L}-\mathrm{V}$ o V-L) y de 0 a cada secuencia repetida (L-L o V-V). El
I.S se obtuvo dividiendo la suma de secuencias entre el total de secuencias. Por ejemplo, si la secuencia temporal de TA reportadas en un partido fue $\mathrm{L}-\mathrm{V}-\mathrm{V}-\mathrm{L}-\mathrm{L}$, los valores asignados serían: $1-0-1-0, \quad$ I.S $=2 / 4=0.50$. Los casos en que se sancionaron dos tarjetas simultáneamente, una para cada equipo, se identificaron como S-S y no se contabilizaron como secuencia con respecto a la decisión anterior ni posterior pero si entre ellas (p.ej. LV-S-S-L, se contó la secuencia entre L y V, y entre $\mathrm{S}$ y $\mathrm{S}$, pero no entre $\mathrm{V}$ y $\mathrm{S}$ ni entre $\mathrm{S}$ y $\mathrm{L}$, así: $1-1, \quad \mathrm{IS}=2 / 2=1.00)$.

Tabla 1. Estadística descriptiva por variable

\begin{tabular}{|c|c|c|c|c|c|c|}
\hline \multirow[b]{2}{*}{ Variable } & \multirow[b]{2}{*}{ Categorías } & \multirow[b]{2}{*}{$\mathrm{N}$} & \multicolumn{2}{|c|}{ Tarjetas Amarillas } & \multicolumn{2}{|c|}{ Tarjetas Rojas } \\
\hline & & & Prom & DS & Prom & DS \\
\hline \multirow[t]{2}{*}{ División } & $1^{\mathrm{a}}$ & 343 & 5.83 & 2.16 & 0.78 & 0.96 \\
\hline & $2^{a}$ & 232 & 5.63 & 2.13 & 1.04 & 1.09 \\
\hline \multirow[t]{2}{*}{ Condición } & Local & 575 & 2.73 & 1.34 & 0.35 & 0.59 \\
\hline & Visitante & 575 & 3.02 & 1.43 & 0.46 & 0.68 \\
\hline \multirow[t]{3}{*}{ Experticia } & FIFA & 253 & 5.77 & 2.18 & 0.80 & 1.00 \\
\hline & $1^{\circ} \operatorname{div}$ & 204 & 5.82 & 2.17 & 0.94 & 0.93 \\
\hline & $2^{\circ} \operatorname{div}$ & 118 & 5.58 & 2.04 & 0.97 & 1.19 \\
\hline \multirow[t]{2}{*}{ Periodo } & $1^{\mathrm{a}}$ tiempo & 536 & 2.51 & 1.55 & 0.17 & 0.43 \\
\hline & $2^{\mathrm{a}}$ tiempo & 536 & 3.29 & 1.79 & 0.63 & 0.88 \\
\hline \multirow[t]{9}{*}{ Total goles } & 0 & 31 & 5.61 & 2.25 & 0.39 & 0.76 \\
\hline & 1 & 84 & 5.40 & 2.15 & 0.60 & 0.66 \\
\hline & 2 & 110 & 5.55 & 2.19 & 0.78 & 0.92 \\
\hline & 3 & 119 & 5.80 & 2.14 & 0.80 & 0.97 \\
\hline & 4 & 105 & 6.01 & 2.14 & 1.05 & 1.10 \\
\hline & 5 & 68 & 5.93 & 1.95 & 0.94 & 1.06 \\
\hline & 6 & 36 & 5.97 & 2.16 & 1.19 & 0.98 \\
\hline & 7 & 15 & 5.73 & 2.74 & 2.27 & 1.49 \\
\hline & 8 o más & 7 & 6.14 & 1.77 & 2.14 & 1.35 \\
\hline \multirow{10}{*}{$\begin{array}{c}\text { Gol } \\
\text { diferencia }\end{array}$} & -4 & 152 & 5.77 & 2.35 & 0.78 & 1.00 \\
\hline & -3 & 123 & 5.80 & 2.03 & 0.82 & 0.98 \\
\hline & -2 & 74 & 5.93 & 1.99 & 1.00 & 1.03 \\
\hline & -1 & 43 & 5.26 & 2.24 & 0.86 & 0.99 \\
\hline & 0 & 17 & 5.53 & 1.70 & 1.29 & 1.21 \\
\hline & 1 & 14 & 5.21 & 2.08 & 1.43 & 0.94 \\
\hline & 2 & 92 & 5.96 & 2.21 & 0.84 & 1.06 \\
\hline & 3 & 31 & 5.74 & 2.08 & 1.03 & 0.95 \\
\hline & 4 & 18 & 5.67 & 1.94 & 0.78 & 0.94 \\
\hline & 5 o más & 11 & 5.09 & 1.92 & 0.80 & 1.27 \\
\hline
\end{tabular}




\section{Análisis de datos}

Después de la estadística descriptiva, se realizaron seis Anovas de una vía para cada uno de los juicios (TA y TR), en función de las siguientes variables: a) división $\left(1^{\mathrm{a}}\right.$ o $\left.2^{\mathrm{a}}\right)$; b) experticia entendida como cualificación o nivel de desempeño (árbitros FIFA, árbitros autorizados para dirigir en $1^{\mathrm{a}}$ división, o árbitros autorizados para dirigir en $2^{\mathrm{a}}$ división); c) total de goles (número total de goles marcados durante el partido); d) gol diferencia (diferencia de goles en el marcador final entre local y visitante); e) periodo ( $1^{\circ}$ tiempo o $2^{\circ}$ tiempo); y f) árbitro (en total 68 quienes fueron codificados para conservar la confidencialidad).

Sobre los partidos únicamente de la $1^{\mathrm{a}}$ división, se realizaron dos Anovas de dos vías, para cada uno de los juicios (TA y TR): equipo por condición $(13 \times 2)$ y equipo por árbitro (19x2). Adicionalmente, se realizaron otros dos Anovas de una vía sobre el efecto de secuencia para TR y para TA, en función del árbitro. Por último, se realizó un Anova de tres vías (condición $\mathrm{x}$ equipo $\mathrm{x}$ árbitro) para TA y para TR.

\section{RESULTADOS}

Para el análisis por periodo de tiempo, se excluyeron los partidos cuyos informes no presentaban el minuto al que se produjo la sanción, reduciéndose la muestra a 536.Para la valoración de la experticia, entendida más como calificación o nivel que como años de experiencia, se definieron tres categorías: árbitros internacionales reconocidos por la FIFA, árbitros autorizados por la Comisión de Arbitraje nacional para dirigir en $1^{\mathrm{a}}$ División, o aquellos autorizados para hacerlo sólo en $2^{\mathrm{a}}$. El total de goles marcados corresponde al número final de goles anotados por partido, mientras que el total de goles diferencia se refiere al marcador final con respecto al equipo local (p.ej. -2 equivale a un resultado en contra del local por dos goles de diferencia, mientras que 2 correspondería a un resultado similar pero a favor del local). La estadística descriptiva obtenida para cada variable en función de las dos variables dependientes, TA y TR, se presenta en la Tabla 1.

Los ANOVAS simples señalaron diferencias significativas entre $1^{\mathrm{a}}$ y $2^{\mathrm{a}}$ División para TR siendo mayor el número de sanciones impuestas en los partidos de $2^{\mathrm{a}}(\mathrm{p}=0.003)$. La cantidad de TA y TR fue significativamente mayor en el segundo tiempo en comparación con el primero ( $p=0.001$ y 0.000 respectivamente). Los equipos en condición de visitante recibieron mayor número de amonestaciones y expulsiones que las recibidas en condición de local ( $\mathrm{p}=0.004$ y 0.002 respectivamente). No se hallaron diferencias estadísticas en ninguna de las variables dependientes en función de la experticia de los jueces (Figuras 1, 2 y 3 ).

\section{Figura 1. Promedios de TA y TR, por División}

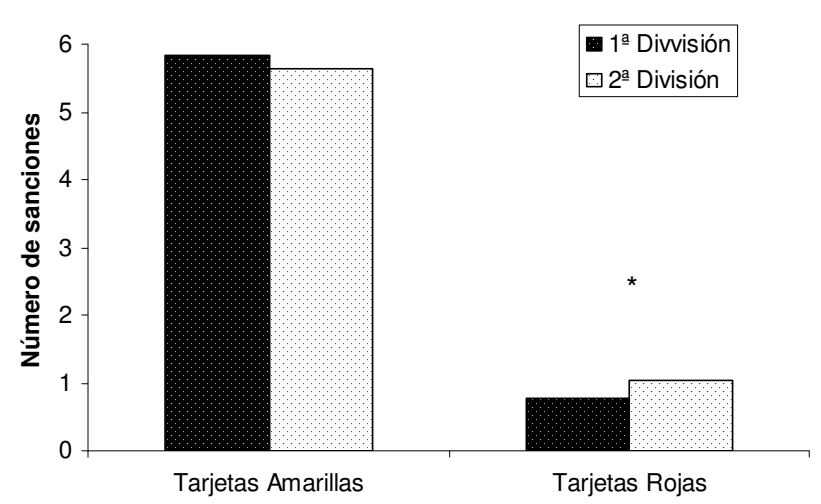

Figura 2. Promedios de TA y TR, para el equipo local y para el visitante

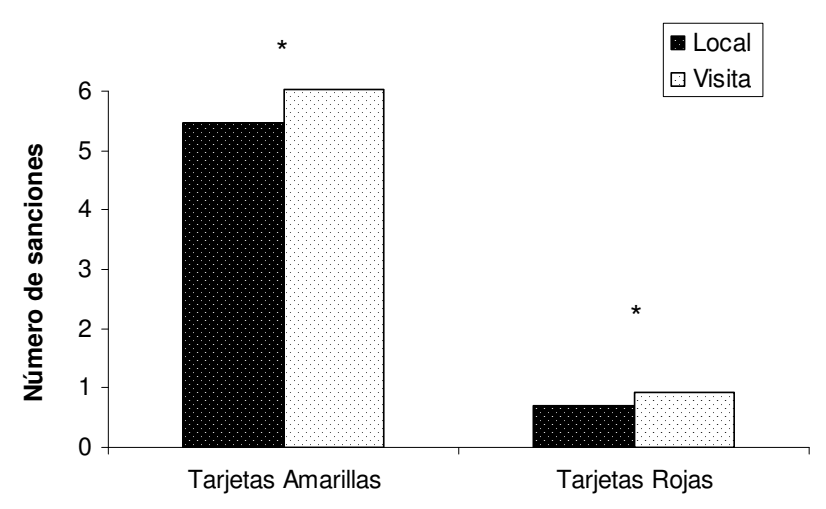


Figura 3. Promedios de TA y TR, por tiempo de partido

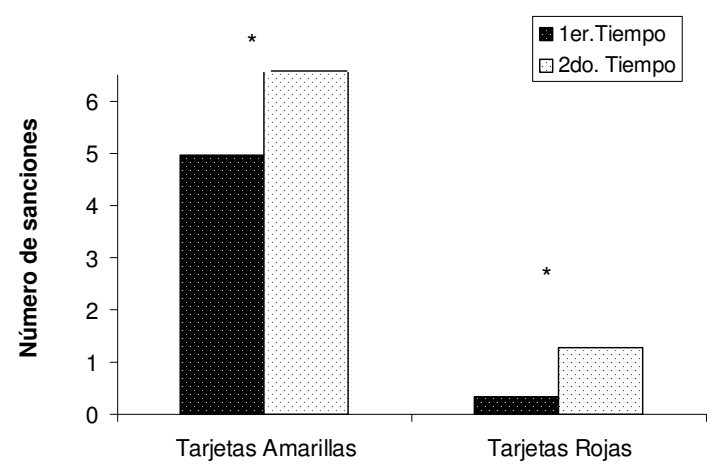

Con respecto al total de goles anotados, no hubo diferencias en TA pero sí en TR $(\mathrm{p}=0,000)$; el análisis posthoc reveló que las diferencias significativas se presentaron entre 0 y 1 con respecto a $4,6,7$ y 8 , entre 2,3 y 5 con respecto a 7 y 8 , y entre 4 y 6 con respecto a 7 (Figura 4). Aunque no se obtuvieron diferencias significativas con respecto al número de goles de diferencia, se pudo observar un leve incremento en el número de TR cuando el marcador es mas parejo entre los equipos, contrario a lo que sucede con las TA con las que se observa una ligera alza a medida que el marcador es mas amplio a favor de cualquiera de los equipos.

\section{Figura 4. TR según número total de goles} marcados

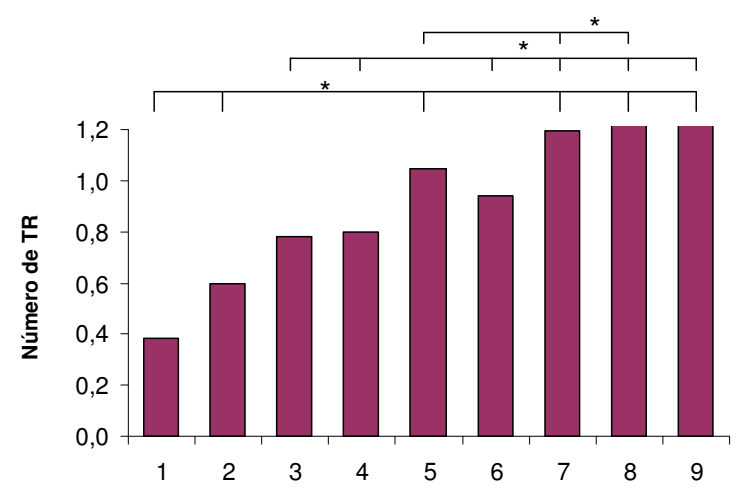

No se encontraron diferencias ni para TA ni para TR con respecto a los equipos (13 de $1^{a}$ división y 22 de $2^{a}$ ), y como tendencia sólo se observó una menor cantidad de TA para el equipo Limonense, el cual jugó en $1^{\mathrm{a}}$ división la temporada 2001-2002 al final de la cual descendió a la $2^{\mathrm{a}}$; su lugar para el torneo 2002-2003 fue ocupado por el equipo de Guanacaste.

Con respecto a los árbitros, se hallaron diferencias significativas en el número de TA sancionadas al equipo local $(\mathrm{p}=0,000)$ y mediante el análisis post hoc se determinaron las diferencias entre ellos. Se identificaron cinco árbitros que sancionan significativamente más TA contra el equipo local, y dos que sancionan más en contra del equipo de visita, en comparación con los árbitros que mostraron la menor frecuencia. Con respecto a las sanciones de TR, dos árbitros dictaminaron un mayor número de éstas en contra del equipo visitante, mientras que no hubo diferencias significativas entre los árbitros en cuanto al número de TR sancionadas en contra del equio local

En segunda instancia, se procedió a hacer un análisis mas específico solamente sobre los partidos de $1^{\text {a }}$ División; la muestra se redujo a 278 partidos, 13 equipos y 19 árbitros. Se observaron resultados similares a los obtenidos con la muestra total: menos sanciones para el equipo local $(\mathrm{p}=0,004$ para TA y 0,002 para TR), mayor número de sanciones en el segundo tiempo con respecto al primero $(\mathrm{p}=0,003$ para TA y 0,001 para TR). La estadística descriptiva se presenta en la Tabla 2

Para el análisis del efecto de secuencia se eliminaron los partidos en los que hubo menos de dos TA o TR, así como aquellos cuyos informes no contenían el reporte del minuto de la sanción ( $\mathrm{N}=19$ para IS-TA y $\mathrm{N}=13$ para IS-TR). El anova para IS-TA no dio significativo mientras que para las TR dio una significancia de $p=0,001$. Tres árbitros presentaron un IS significativamente mayor que el IS obtenido por los de IS menor; sin embargo el IS estuvo por encima de 0,5 tanto para las TA como para la TR, algo más alto éste último pero también con mayor variabilidad (Tabla 3 ). 
Tabla 2. Estadística descriptiva de partidos de $1^{\text {a }}$ división. para cada variables

\begin{tabular}{|c|c|c|c|c|c|c|}
\hline \multirow[b]{2}{*}{ Variable } & \multirow[b]{2}{*}{ Categorías } & \multirow[b]{2}{*}{$\mathrm{N}$} & \multicolumn{2}{|c|}{$\begin{array}{c}\text { Tarjetas } \\
\text { Amarillas }\end{array}$} & \multicolumn{2}{|c|}{$\begin{array}{c}\text { Tarjetas } \\
\text { Rojas }\end{array}$} \\
\hline & & & Prom & DS & Prom & DS \\
\hline \multirow[t]{2}{*}{ Condición } & Local & 278 & 2.79 & 1.29 & 0.31 & 0.52 \\
\hline & Visita & 278 & 3.14 & 1.44 & 0.48 & 0.71 \\
\hline \multirow[t]{13}{*}{ Equipo } & Carmelita & 45 & 2.82 & 1.48 & 0.47 & 0.76 \\
\hline & Cartaginés & 46 & 3.17 & 1.22 & 0.52 & 0.78 \\
\hline & Guanacaste & 16 & 3.50 & 1.55 & 0.38 & 0.62 \\
\hline & Herediano & 48 & 3.19 & 1.42 & 0.44 & 0.68 \\
\hline & Liga D.A. & 51 & 2.76 & 1.45 & 0.33 & 0.51 \\
\hline & Liberia & 53 & 2.96 & 1.19 & 0.36 & 0.56 \\
\hline & Limonense & 31 & 2.39 & 1.15 & 0.55 & 0.81 \\
\hline & Osa & 42 & 2.95 & 1.40 & 0.36 & 0.58 \\
\hline & Pérez Zeledón & 40 & 2.85 & 1.56 & 0.25 & 0.54 \\
\hline & San Carlos & 42 & 3.07 & 1.47 & 0.31 & 0.47 \\
\hline & Santos & 49 & 3.04 & 1.38 & 0.41 & 0.64 \\
\hline & Saprissa & 42 & 2.86 & 1.05 & 0.33 & 0.53 \\
\hline & Sta Bárbara & 50 & 3.10 & 1.47 & 0.46 & 0.68 \\
\hline
\end{tabular}

Tabla 3. Indice de secuencia en TA y en TR por árbitro

\begin{tabular}{|c|c|c|c|c|c|c|}
\hline \multirow[b]{2}{*}{ Arbitro } & \multirow[b]{2}{*}{$\mathrm{N}$} & \multicolumn{2}{|c|}{$\begin{array}{c}\text { Tarjetas } \\
\text { Amarillas }\end{array}$} & \multirow[b]{2}{*}{$\mathrm{N}$} & \multicolumn{2}{|c|}{ Tarjetas Rojas } \\
\hline & & $\begin{array}{c}\text { IS en } \\
\text { TA }\end{array}$ & DS & & $\begin{array}{c}\text { IS en } \\
\text { TR }\end{array}$ & DS \\
\hline 1 & 14 & 0.58 & 0.28 & 7 & 0.86 & 0.24 \\
\hline 2 & 8 & 0.57 & 0.15 & 7 & 0.71 & 0.49 \\
\hline 3 & 12 & 0.59 & 0.20 & 7 & 0.71 & 0.49 \\
\hline 4 & 16 & 0.59 & 0.30 & 7 & 0.86 & 0.38 \\
\hline 5 & 10 & 0.45 & 0.23 & 5 & 1.00 & 0.00 \\
\hline 6 & 16 & 0.63 & 0.24 & 6 & 0.83 & 0.26 \\
\hline 7 & 19 & 0.58 & 0.24 & 6 & 0.42 & 0.38 \\
\hline 8 & 15 & 0.48 & 0.32 & 6 & 0.82 & 0.40 \\
\hline 9 & 9 & 0.65 & 0.24 & 6 & 0.17 & 0.41 \\
\hline 10 & 8 & 0.64 & 0.31 & 7 & 0.21 & 0.39 \\
\hline 11 & 28 & 0.56 & 0.21 & 7 & 0.71 & 0.49 \\
\hline 12 & 21 & 0.61 & 0.23 & 5 & 0.30 & 0.27 \\
\hline 13 & 9 & 0.60 & 0.19 & 5 & 0.83 & 0.24 \\
\hline 14 & 8 & 0.61 & 0.31 & & & \\
\hline 15 & 22 & 0.62 & 0.23 & & & \\
\hline 16 & 24 & 0.52 & 0.23 & & & \\
\hline 17 & 12 & 0.54 & 0.22 & & & \\
\hline 18 & 8 & 0.44 & 0.20 & & & \\
\hline 19 & 17 & 0.45 & 0.14 & & & \\
\hline
\end{tabular}

No se halló ninguna interacción significativa ni en los anovas de dos vías (equipo $\mathrm{x}$ condición, equipo $\mathrm{x}$ árbitro, $\mathrm{ni}$ condición $\mathrm{x}$ árbitro), tampoco en el anova de tres vías, equipo x condición $\mathrm{x}$ árbitro). Sólo se ratificó el efecto simple significativo de la condición y del árbitro.

Retomando el objetivo de esta investigación, cual fue el determinar si existen tendencias entre los árbitros de fútbol en sus juicios sobre amonestaciones y expulsiones, es importante recalcar que el presente estudio se limitó a medir el número de sansiones disciplinarias impuestas por los árbitros, sin evaluar la pertinencia de las mismas dado que la metodología empleada no permite discriminar entre juicios correctos y juicios incorrectos; no se tomaron en consideración otras juicios deportivos, como penas máximas, tiros libres directos o indirectos, juicios de fuera de lugar, u otros, que igualmente pueden resultar decisivos en algun momento $y$ circusntancia particular de un partido. El mayor o menor número de sanciones disciplinarias impuestas en un partido, depende tanto de la conducta de los jugadores como del desempeño de los árbitros quienes ademas de poseer una preparación particular, poseen sus propios esquemas cognitivos. Bar-Eli, LevyKalker, Bridwell y Goodwin (1999) comprobaron que el comportamiento de los jugadores en el campo de juego está influido por su conocimiento de las expectativas relativas a la tolerancia de los árbitros hacia el equipo local y tales expectativas inciden en su comportamiento intencional táctico $\mathrm{y}$ estratégico.

Resumiendo los resultados, no se encontraron diferencias significativas con respecto a las variables experticia, goles de diferencia entre el equipo local y el visitante aunque se observaron tendencias diferentes entre TA y TR, ni equipo. Se hallaron diferencias significativas para el número de TR según la División (mayor número en la $2^{\mathrm{a}}$ que en la $1^{\text {a }}$ ) y el total de goles marcados (a mayor número de goles, mayor número de TR). Para las demás variables, se hallaron diferencias tanto para TA como para TR ( periodo, árbitro y condición)

En cuanto al fenómeno de la ventaja de local, los resultados son consistentes con lo reportado por diferentes autores (Nevill, Newell y Gale, 1996; FIFA, 2001; Jones, Bray y Bolton, 2001; Nevill, Balmer y Williams, 2002); como se anotaba anteriormente, el 
mecanismo que explica tal tendencia no ha sido aun satisfactoriamente esclarecido; al respecto la FIFA (2001) afirma que a pesar de los numerosos estudios sobre el mito de la ventaja local, los resultados sobre su mecanismo son contradictorios (la mayoría centrados en el público), pero resultan insuficientes para explicar los factores que determinan el fenómeno.

Así mismo, el mayor número de sanciones observadas en el segundo tiempo de partido con respecto al primero, tanto para las TA como para las TR, apoyan los hallazgos de Bar-Ely y Tractinsky (2000) y de Segura y cols. (1999) quienes hallaron que durante la etapa final de un partido de baloncesto se incrementa el número de jugadas críticas y la percepción de conflicto por parte de los árbitros.

Contrario a los hallazgos de anteriores investigaciones (Paull y Glencross, 1997; Kioumourtzoglou, Kourtesis, Michaelopolou y Derri, 1998; Segura y cols., 1999; Vasconcelos y Carvalho, 2000; MacMahon y Ste-Marie, 2002), en este no se encontró relación entre juicios y experticia de los árbitros, posiblemente debido a los diferentes criterios de categorización de la variable o al mecanismo de medición de la variable dependiente; se podría interpretar también como una homogeneidad en el nivel de preparación y de destreza del colectivo arbitral

Frente a la diferencia entre $1^{\mathrm{a}}$ y $2^{\mathrm{a}}$ División, los resultados coinciden parcialmente con los reportados por Torregrosa y Cruz quienes hallaron que en la $2^{\mathrm{a}}$ se presenta mayor número de protestas y de juego brusco por parte de los jugadores de fútbol, aunque ellos sólo encontraron diferencias significativas con respecto a las TA y no con las TR, como se encontró en el presente.

Las tendencias en los juicios arbitrales se observaron de manera mas clara frente a las sanciones de expulsiones que frente a las de amonestaciones. Fue en el caso de las TR en que se hallaron mas diferencias estadísticamente significativas, lo cual es coincidente con lo reportado por Plessner y Betsch quienes no hallaron efecto de secuencia para los juicios de tiros libres pero sí para los de penas máximas. Lo anterior tiene su explicación sobre la base de la trascendencia de la decisión tomada. Según Bereby-Mayer y cols. (2003) cuando las consecuencias de una decisión son mas trascendentales la reacción que genera su decisión es mas intensa y por tanto el proceso de toma de decisión por parte del actor es diferente frente a estas que frente a decisiones de menor trascendencia. Los mismos autores afirman que la decisión de no emitir respuesta es mas probable cuando no hay consecuencia negativa; ellos encontraron una tasa diferencial de omisiones que sigue el patrón predicho por la teoría de las probabilidades: los sujetos presentan mayor número de omisiones en las situaciones en que no se anticipaba consecuencia negativa por no responder, con respecto a las situaciones en las que se establecía consecuencia negativa. En el fútbol la consecuencia de sancionar una expulsión conlleva una consecuencia mas negativa que el no hacerlo, lo cual podría explicar por qué el reducido número de TR frente al elevado número de TA, las cuales no conllevan consecuencias importantes para el árbitro.

La atribución de responsabilidad por los juicios arbitrales recaen solo sobre la persona del árbitro por lo que de acuerdo con Zeelemberg y cols. (2000), la reacción afectiva post-decisión se caracteriza por ser generalmente intensa, como de hecho se observa con frecuencia al finalizar un partido en el que una decisión del árbitro ha sido decisiva en el resultado final del encuentro. Cabría entonces preguntarse si es mas fácil para el árbitro decidir no actuar, en este caso, decidor no sancionar una tarjeta roja por el costo de reacción que ella implica. De hecho los resultados parecen indicar que esta tendencia la presentan algunos árbitros, a quienes $\mathrm{s}$ eles podría denominar "condescendientes".

Con respecto al efecto de secuencia comprobado por Plessner y Betsch (2001), los resultados apoyan los resultados previos. En un rango de 0 a 1 , los promedios tanto pata TA como para TR se ubican en la mitad superior (0,56 y 0.65 respectivamente), valores que pueden interpretarse como medios; sin embargo el rango de variabilidad fue mayor para las TR en las que sí se encontró 
diferencias significativas entre algunos árbitros. En este aspecto, se podría decir que algunos árbitros son "compensadores", mientras que otros son "localistas" o "antilocalistas", pues hubo árbitros en quienes la diferencia entre sanciones al equipo local y al equipo visitantes fue significativa, unos a favor del primero, otros a favor del segundo.

En conclusión, se comprueba la existencia de algunas tendencias que sesgan los juicios de los árbitros de manera diferencial (localía y efecto de secuencia). Complementariamente, se identifican algunas características deportivas que se relacionan de manera diferencial con el número de sanciones disciplinarias que se imponen en un partido (división, marcador, periodo).

Tales tendencias individuales subyacen inevitablemente a la tarea de interpretar una acción, por lo que la medida a tomar para disminuir o atenuar su efecto sobre el desempeño arbitral no debería orientarse a su eliminación, sino mas bien a su reconocimiento por parte de cada uno de los árbitros. Puede suceder que la mism persona no sea consciente de sus propios sesgos, o que la información con la que se le juzga presenta el mismo sesgo de subjetividad por el que se le cuestiona. El presentar una información objetiva, puede promover un proceso de autoevaluación que a la vez conduzca a mejorar el propio desempeño.

Es importante resaltar que no se encontró evidencia que apoye la creencia general de que los árbitros están comprometidos con un equipo particular, compromiso que los lleva a favorecer con sus juicios al equipo en cuestión o a perjudicar al rival. No se halló ninguna relación significativa estadísitcamente entre árbitro y equipo.

Por último, los resultados obtenidos en el presente estudio no son concluyentes en tanto sólo señalan la relación entre algunas variables deportivas y las sansiones disciplinarias, pero no explica las tendencias identificadas, lo cual puede llevar a conclusiones equivocadas. Por lo tanto, se requiere proseguir en esta línea con investigaciones de nivel explicativo, que permitan además de determinar la causaliudad de tales tendencias, discirminar entre juicios correctos y juicios incorrectos.

\section{Referencias}

Alain, C., Sarrazin, C. y Lacombe, D. (1986). The use of subjetive expected values in decision-making in sport. En: D.M. Landers (Ed.). Sport and elite performers (pp.1-6). Champaign, IL: Human Kinetics.

Ansorge, C. y Scheer, J. (1988). International bias in judging gymnastic competition at the 1984 olympic games. Research Quarterly, 59(2), 103-107.

Bar-Ely, M. y Tractinsky, N. (2000). Criticality of game situations and decision making in basketball: an application of performance crisis perspective. Psychology of Sport and Exercise, 1(1), 27-39.

Bereby-Meyer, Y., Meyer, J. y Budescu, D. (2003). Decision making under internal uncertainty: the case of multiple-choice test with different scoring rules. Acta Psychologica, 112(2), 207-220.

Caracuel, J.C., Andreu, R. y Pérez, E. (1995). Análisis psicológico del arbitraje y juicio deportivos: una aproximación desde el modelo interconductual. Motricidad, 1, 5-24.

FIFA (2001). La gran ventaja de los partidos en casa. FIFA Magazine, febrero, 7-10.

Jentzsch, I. y Sommer, W. (2002). The effect of intentional expectancy on mental processing: a chronopsychophysiological investigation. Acta Psychologica, 111(3), 265-282.

Jones, M.V., Bray, S.R. y Bolton, L. (2001). Game location and officiating bias in English Club Cricket. Perceptual and Motor Skillls, 93(2), 359362.

Jones, M.V., Paull, G.C. y Erskine, J. (2002). The impact of a team's aggressive reputation on the decisions of association football referees. Journal of Sports Sciences, 20(12), 991-1000.

Kioumourtzoglou, E., Kourtessis, T., Michalopoulou, M. y Derri, V. (1998). Differences in several perceptual abilities between experts and novices in basketball, volleyball and water-polo. Perceptual and Motor Skills, 86(3 Part 1), 899-912.

Landers, D.M. (1970). A review of research on gymnastics judging. Journal of Health, Physical Education and Recreation, 14, 85-88.

MacMahon, C. y Ste-Marie-D. (2002). Decision making by experienced rugby referres: use of perceptual information and episodic memory. Perceptual and Motor Skills, 95(2), 570-573.

MacMahon, C. y Ste-Marie-D. (2002). Decision making by experienced rugby referres: use of perceptual information and episodic memory. Perceptual and Motor Skills, 95(2), 570-573.

Nevill, A.M., Balmer, N.J. y Williams, M.A. (2002). The influence of crowd noise and experience upon refereeing decisions in football. Psychology of Sport and Exercise, 3(4), 261-273. 
Nevill, A.M., Newell, S.M. y Gale,S. (1996). Factors associated with home advantage in English and Scottish soccer matches. Journal of Sports Sciences, 14 (2), 181-186.

Paull, G. y Glencross, D. (1997). Expert perception and decision making in baseball. International Journal of Sport Psychology, 28(1), 35-56.

Plessner, H. y Betsch, T. (2002). Refereeing in sports is supposed to be a craft, not an art: response to Mascarenhas, Collins y Mortimer (2002). Journal of Sport and Exercise Psychology, 24(4), 334-337.

Plessner, H.(1999). Expectation biases in gymnastics judging. Journal of Sport and Exercise Psychology, 21(2), 131-144.

Roelofsma, P.H.M.P. y Van der Pligt, J. (2001). On the psychology of time preference and human decisions: introduction to the special issue. Acta Psychologica, 108(2), 91-93.

Scheer, J. (1973). Effect of placement in the order of competition on scores of Nebraska high school students. Research Quarterly, 44(1), 79-85.

Segura, J. y cols. (1999). Toma de decisiones y experiencia en árbitros de baloncesto. En: Sociedad Murciana de Psicología de la Actividad Física y el Deporte.(Ed.) VII Congreso Nacional de Psicología de la Actividad Física y el Deporte. Memorias (pp. 642-655). Murcia: Autor.

Torregrosa, M. y Cruz, J. (1997). Diferencias de arbitraje entre la liga de fútbol profesional (LFP) y la premier league (PL): cuestión de cantidad o de calidad? En: F. Guillén (Ed.). La psicología del deporte en España al final del milenio. Actas del VI Congreso Nacional de Psicología de la Actividad Física y el Deporte. (pp.575-582). Las Palmas: Universidad de Las Plamas de Gran Canaria.

Vasconcelos-Raposo, J.J.B. y Carvalho, R. (2000). Ansiedad cognitiva, somática y autoconfianza en árbitros de balónvolea. Revista de Psicología del Deporte, 9 (1-2), 135-142.

Vasconcelos-Raposo, J.J.B. y Carvalho, R. (2000). Ansiedad cognitiva, somática y autoconfianza en árbitros de balónvolea. Revista de Psicología del Deporte, 9 (1-2), 135-142.

Zeelemberg, M., Van der Pligt, J. y De Vries, N. (2000). Attributions of responsability and effective reactions to decision outcomes. Acta Psychologica, 104(3), 303-315. 